\title{
The paradox of increasing mass
}

\author{
Alexandre GEORGES ${ }^{1}$ \\ ${ }^{1}$ office@ $@$ professeur-alexandre-georges.info
}

\begin{abstract}
As demonstrated by Pound-Rebka experiment, the wave period of an electromagnetic radiation can be contracted or dilated due to the deformation of the geometry of Space-Time. In cosmology, this principle is the explanation of the phenomenon of gravitational redshift, highlighting an expansion of the universe caused by space dilation. One of the consequences of this fact is a kind of rupture of energy conservation, directly induced by the Planck-Einstein relation, which would then be a relative conservation of energy. In this paper, this phenomenon will be extended to mass particles, by the applying De Broglie's thesis, which will pose a mass conservation paradox. This paradox will show that mass is, like energy, relative to the deformation of the geometry of space-time in which the object is situated. No sufficient solution will be made here to this paradox.
\end{abstract}

\section{Introduction}

Pound-Rebka experiment has demonstrated the influence of the deformation of the space-time geometry on the wave period of electromagnetic radiation [2] [3]. It is this fact that is the cause of the phenomenon of gravitational redshift observed highlighting an expansion of the Universe due to the expansion of space. Thus, the amount of relative energy associated with the wave, by the Plack-Einstein relation:

$$
E=h v
$$

Varies according to the deformation of the space-time in which the photon is located:

$$
\begin{gathered}
E=h v \\
E=\frac{h}{d \tau} \\
E=\frac{h}{d t \sqrt{1-\frac{\frac{2 G M}{c^{2}}}{r}}}
\end{gathered}
$$

Here, we have extracted the expression of the proper time of the Schwarzschild metric [1], a weak field approximation for mass, immobile, non-charged, non-rotating, and spherical space objects:

$$
d s^{2}=\left(1-\frac{2 G M}{c^{2} r}\right) c^{2} d t^{2}-\left(1-\frac{2 G M}{c^{2} r}\right)^{-1} d r^{2}-r^{2}\left(d \theta^{2}+\sin ^{2} \theta d \varphi^{2}\right)
$$

This is a break in the conservation of energy, which could be described as a relative conservation of energy, since the energy level of the radiation does not vary without reason, but relatively to the space-time curvature [4]. 
Thus, a photon $\gamma$ of energy $E_{1}$ at time 1, moving towards an $\Omega$ object (non-rotating, motionless, and spherical space), of mass $M$, would have an energy $E_{2}$ at time 2, satisfying, for inequality:

$$
r_{1}>r_{2}
$$

That:

$$
E_{1}<E_{2}
$$

From the point of view of an observer located in an almost flat space-time.

\section{Application to mass subatomic particles}

In application of De Broglie's thesis, we can express:

$$
\lambda=\frac{h}{m v}
$$

In order to distinguish, later, the relativistic influence of the speed variations and of space-time curvature variations on the wavelength of the particle, we integrate by default the Lorentz factor at the wavelength, which is then a relative wavelength.

We will use the same $\Omega$ object of mass $M$ as in the previous section, but replace $\gamma$ by a particle $\varepsilon$ of mass $m_{1}$ at time 1, heading towards the Omega object. An observer located in an almost flat space-time observe the relative wavelength $\Theta$.

Considering the following development:

$$
\begin{gathered}
\lambda=\frac{h}{m v} \\
\Theta=\frac{h}{m v^{2}} \\
\theta \sqrt{1-\frac{\frac{2 G M}{c^{2}}}{r}}=\frac{h}{m v^{2}}
\end{gathered}
$$

Where $\theta$ is the period relative to the speed, but not to the space-time curvature.

$$
m=\frac{h}{v^{2} \theta \sqrt{1-\frac{\frac{2 G M}{c^{2}}}{r}}}
$$

We can then state that inequality:

$$
m_{1}<m_{2}
$$


Is verified for inequality:

$$
r_{1}>r_{2}
$$

From the point of view of an observer located in an almost flat space-time.

We have not taken into account non-relativistic phenomena, such as velocity variations due to the acceleration of the falling $\varepsilon$ particle towards the $\Omega$ object.

This inequality of mass $m_{1}$ and $m_{2}$ constitutes a certain problem. Indeed, if our particle was an electron, then we would have an electron of variable mass, here increasing. This would be a problem different from that of relative mass, a concept induced by Restricted Relativity.

In particle physics, the paradox is then:

A $\beta$ electron of mass $m$, which would gravitate around its $\alpha$ nucleus of mass $M$, would give the latter a mass gain due to the deformation of the geometry of space-time caused by its own mass. At the same time, the deformation of the space-time geometry caused by $M$ of $\alpha$ would cause an increase of $m$ of $\beta$.

However, if $M$ increases because of the space-time curvature caused by $m$, then the curvature caused by $M$ is greater than initially and increases $m$, etc.

In the same way, if $m$ increases because of the space-time curvature caused by $M$, then the curvature caused by $m$ is greater than initially and $M$ is still growing, etc.

Here, we have not only an increase of mass depending on the gravitational bath in which the particle is located, we obtain an atom of increasing mass, according to the system of equations:

$$
\left\{\begin{array}{l}
m_{n+1}=\frac{h}{v^{2} \theta \sqrt{1-\frac{\frac{2 G M_{n}}{c^{2}}}{r}}} \\
M_{n+1}=\frac{h}{v^{2} \theta \sqrt{1-\frac{\frac{2 G m_{n}}{c^{2}}}{r}}}
\end{array}\right.
$$

Where, for the sake of simplicity, we used:

$$
d \tau=d t \sqrt{1-\frac{\frac{2 G M}{c^{2}}}{r}}
$$

From:

$$
d s^{2}=\left(1-\frac{2 G M}{c^{2} r}\right) c^{2} d t^{2}-\left(1-\frac{2 G M}{c^{2} r}\right)^{-1} d r^{2}-r^{2}\left(d \theta^{2}+\sin ^{2} \theta d \varphi^{2}\right)
$$


This is not an approximation that significantly changes conclusions.

In astrophysics, this problem can be extended to the case of galaxies, where each component would then grow the masses of the other components. We would then have a galaxy of increasing mass.

These mass growths only due to a cohabitation in the same local space of objects lead to three problems:

First, the mass of a particle would not be fixed. There is the question of the nature of a particle that evolves in an increasingly intense gravitational field. An electron whose mass would increase would it still be the same particle?

Second, the relative conservation of the mass is no longer, as in the case of energy, only relative to the curvature of the space-time in which the object is situated. It also depends on the duration of coexistence of several mass objects in the same local system. So far, nothing has made it possible to reach this conclusion by observation.

Third, to avoid such a break in the conservation of the mass, it would be necessary to admit, on the one hand, a break of the principle of equivalence mass-energy and, on the other hand, to admit that the deformation of the geometry of the Space-time in which the particle is located can vary the speed of said particle beyond its acceleration due to its fall towards an object. This non-mass mass-particle particle asymmetry does not seem to correspond to what is observed and is not in conformity with General Relativity, from which this paradox originated.

\section{Conclusion}

The development highlighting a paradox of increasing mass has, for the moment, no solution corresponding to the observation or even coherent with the model from which it is derived: General Relativity. This conclusion is therefore not a real conclusion.

In a purely speculative way, we could consider that to apprehend the mass as a consequence of the deformation of space-time would remove from the increase of the relative mass the possibility of really increasing the curvature of space-time. But this is only a hypothetical opening.

Another opening, perhaps more adapted, would be a quantification of space-time providing a solution to this paradox. This is the kind of problem that models like Loop Quantum Gravity will probably have to answer.

In any case, it appears that we have probably not fully understood the nature of spacetime and matter.

\section{References}

[1] K. Schwarzschild, "Über das Gravitationsfeld eines Massepunktes nach der Einsteinschen Theorie", Preussische Akademie der Wissenschaften, Sitzungberichte, 1916, p. 189-196, 1916. 
[2] R. V. Pound and G. A. Rebka, Jr., "Gravitational Red-Shift in Nuclear Resonance", Phys. Rev. Lett., 3, 439, 1959.

[3] R. V. Pound and G. A. Rebka, Jr., "Apparent Weight of Photons", Phys. Rev. Lett., 4, 337, 1960 .

[4] A. Georges, "Incompatibility between Einstein's general relativity and Heisenberg's uncertainty principle", Physics Essays, vol. 31, issue 3, pp. 327-332, 2018.

\section{Additional information}

Corresponding author:

Professor Alexandre GEORGES

Toulouse, France

Contact: office@professeur-alexandre-georges.info

Affiliation:

Projet Energium (private company laboratory registered: 822143 707) - Direction

Bureau du Professeur GEORGES

Contact: direction@ projet-energium.com

Competing interests:

The author declares no competing interests. 